\section{A regenerative medicine}

Axon regeneration after spinal cord injury (SCI) is hampered by the formation of hypertrophic scar tissue and the intrinsically low capacity of CNS axons for regrowth. Attempts to stimulate regeneration by blocking growth-inhibiting factors have met with limited success. Now, Hellal et al. have shown that microtubule

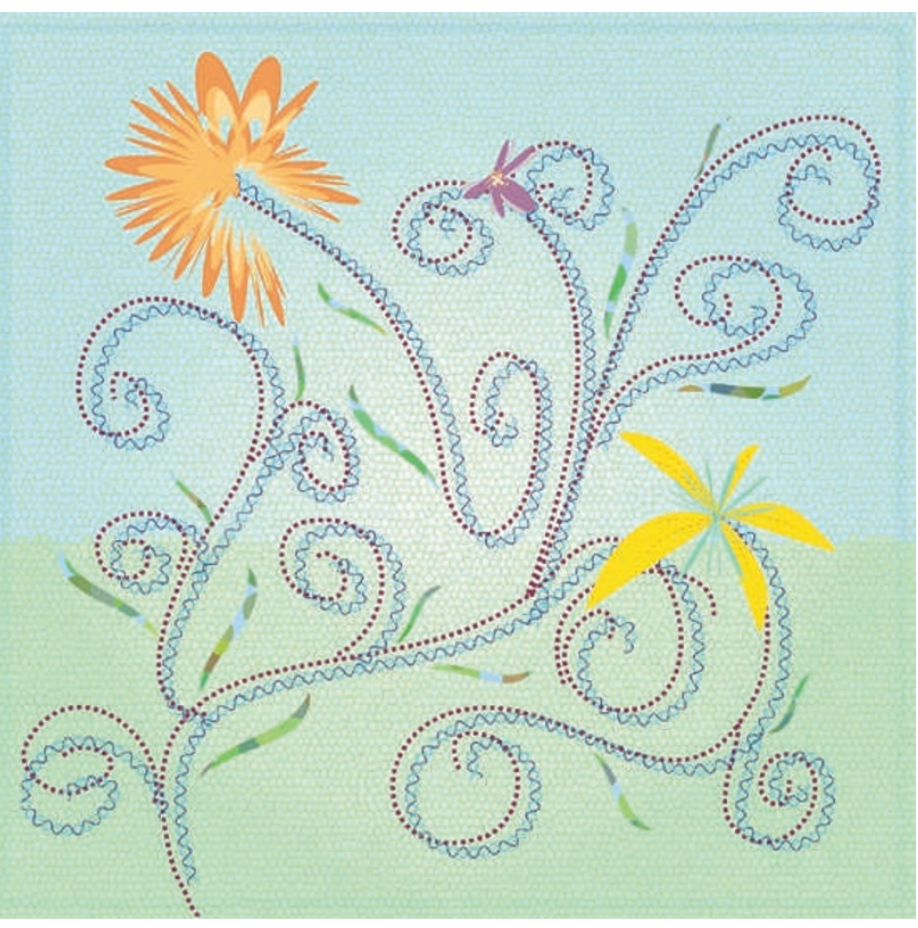

stabilization using paclitaxel can assist repair after SCI.

Microtubules are key components of the cystoskeleton and regulate numerous aspects of scarring. Moreover, lesioned CNS axons have a disorganized microtubule network, and moderate microtubule stabilization is known to promote axon growth in vitro.

The authors performed spinal cord hemisection in rats that then received continuous intrathecal paclitaxel or vehicle. Seven days postinjury, the accumulation of fibrotic proteins such as laminin in the lesion site of vehicle-treated rats was absent in rats that received paclitaxel.

Transforming growth factor- $\beta$ (TGF $\beta$ ) signalling is upregulated following SCI and plays an important part in scar formation. A key downstream mediator is SMAD2, which traffics to the nucleus and controls the expression of genes involved in fibrosis. In the current study, paclitaxel reduced nuclear trafficking of SMAD2 in vivo, and instead caused SMAD2 to localize to microtubules in TGF $\beta$-stimulated astrocytes. The drug also decreased the production of chondroitin sulphate proteogylcans, which inhibit axon growth.

Injury to the peripheral axon of a dorsal root ganglion neuron can produce a growth-competent state in the CNS axon of that neuron provided there is no scarring at the central injury site. In rats treated with paclitaxel following SCI, transection of peripheral axons 2 weeks later led to growth of regenerative fibres at the central lesion site. By contrast, regeneration was absent in vehicle-treated rats. In addition, paclitaxel induced a fivefold increase in the number of serotonergic fibres in the spinal cord below the injury site. Thus, as well as reducing scarring, paclitaxel stimulates the intrinsic growth capacity of neurons.

At the functional level, paclitaxeltreated rats showed improvements in a locomotor test at 6 and 8 weeks post-lesion compared with vehicletreated rats, showing that the cellular effects of paclitaxel were accompanied by physiological effects associated with functional recovery.

These studies highlight the beneficial effects of paclitaxel, an approved anticancer drug, in overcoming the intrinsic barriers to recovery after SCI.

Katie Kingwell

ORIGINAL RESEARCH PAPER Hellal, F. et al. Microtubule stabilization reduces scarring and causes axon regeneration after spinal cord injury. Science 27 Jan 2011 (doi: 10.1126/ science.1201148) 